\title{
A Review on Study and Comparison between 2D Gel Electrophoresis and Mass Spectrometry
}

\author{
Ashwini Yerlekar ${ }^{1}$, Priyanka Dudhe ${ }^{2}$ \\ Department of Computer Technology Yeshwantrao Chavan College of Engineering Nagpur, Maharashtra, India
}

\begin{abstract}
Protein structure prediction is an important area of life sciences. The Protein prophecy is unbreakable because of its complex structure. The main problem in proteomics is to estimate the several proteins available in cell structure or tissue sample. The protein-protein interaction is essential to study the behaviour of protein in a specific environment, and study molecular relationship in living systems. Therefore, large scale proteomics technologies are required to measure physical connection of proteins in living organisms. A combination of high resolution two-dimensional (2-D)gel electrophoresis, highly sensitive biological mass spectrometry, and the speedily growing protein and DNA databases has resulted for high throughput proteomics. This review concentrates on two main techniques of protein identification 2-D gel electrophoresis and High Throughput Mass Spectrometry.
\end{abstract}

Keywords : 2-D gel electrophoresis, DNA database, Mass Spectrometry, Protein prediction, Proteomics

\section{INTRODUCTION}

The term proteomics describes the study and characterization of complete set of proteins present in a cell, organ, or organism at a given time [1]. In general, proteomic approaches can be used (a) for comparative expression analysis of two or more protein samples, (b) for the localization and identification of posttranslational modifications, (c) for proteome profiling, and (d) for the study of protein-protein interactions. Proteome analysis represents large-scale analysis of the proteins in an organism, simultaneously [14], thus facilitating with the elucidation of gene function [15]. The word proteomics was coined by Marc R. Wilkins in 1994, when he was developing the concept as $\mathrm{PhD}$ student in Macquarie University [16]. Proteomics was mainly derived from the field of two-dimensional gel electrophoresis (2-DE). With the arrival of the concept of proteomics or proteome analysis, the method of 2-DE and High throughput Mass Spectrometry have gained greater importance. Although alternative methods of protein separation for proteomics are available, 2-DE has remained the basic technology for protein separation [17]. Proteomics has steadily gained momentum over the past decade with the evolution of several approaches. Few of these are new and others build on already existed methods.2-D gel, LC- Mass spectrometry-based methods and micro arrays are the most common technologies for study of proteins.

There are 2 mass spectrometry based methods currently used for protein expression profiling. The most stable, sustained and easy method uses high resolution, 2 dimensional electrophoresis to differentiate proteins from different samples in parallel, followed by staining and selection of differentially expressed proteins to be identified by mass spectrometry. In spite of the advances in 2DE and its easy understanding, it has its limits as well. The central issue is the inability to predict all the proteins within a sample, given their dramatic range in differing properties and expression level.[19]

The second quantitative approach uses stable isotope tags to different label proteins from two different mixtures. Here, the proteins within a complex mixture are digested to yield labeled peptides before they are tagged isotopically initially. The labeled mixtures are then combined then analyzed by tandem mass spectrometry and peptides are separated by multidimensional liquid chromatography. Isotope coded affinity tag (ICAT) reagents are the largely used isotope tags. Another quantitative approach is the Accurate Mass and Time (AMT) tag approach developed by Smith. In this approach increased throughput and sensitivity is achieved by avoiding the need for tandem mass spectrometry.. Balancing the use of mass spectrometers in proteomics and in medicine is the use of protein micro arrays i.e. Protein Chip. The aim behind protein micro arrays is to analyze thousands of protein scanning features, for the examination of biological samples. Also, arraying of multiple protein types for the study of properties like protein-DNA, protein-protein connections. if possible, the functional proteomic arrays would contain the full sequence of the proteins of a given sample of an organism.

Also, the other technique used is as Reverse Phased Protein Microarrays. This is a promising and newer microarray application for the analysis, study and of complex diseases such as cancer. The technology overlaps the laser capture micro-dissection (LCM) with micro array technology, to produce turn around phase protein microarrays. In this type of microarrays, the whole collection of protein themselves are not mobilized with the goal of capture various stages of disease within an individual patient. 


\section{2-DIMENSIONAL GEL ELECTROPHORESIS TECHNIQUE}

2-Dimensional electrophoresis was first introduced by P. H. O'Farrell [2] and J. Klose [3] in 1975. Within the original method, the first-dimension separation was performed in carrier ampholyte-containing polyacrylamide gels forged in slender tubes [18]. Two-dimensional electrophoresis is a powerful and wide used methodology for the analysis of complicated protein mixtures extracted from different biological samples, tissues or cells. This method type's protein consistent with two freelance properties in two separate steps: the first-dimension step, isoelectric focusing (IEF), separates proteins consistent with their isoelectric points (pI); the second-dimension step, SDS-polyacrylamide gel electrophoresis (SDS-PAGE), separates proteins consistent with their molecular weights. Every spot on the ensuing two-dimensional array corresponds to one protein species within the sample. Thousands of various proteins will therefore be separated, and data like the protein pI, the apparent relative molecular mass, and therefore the quantity of every protein is obtained [18]. The term "electrophoresis" was originally meant to see the migration of charged particles in an electrical field. In other choice term "ionophoresis" had been reserved for the migration of lower relative molecular mass substances in stabilized media like gels and powders. Today, the overall term electrophoresis covers all applications in spite of the fabric being studied and therefore the medium getting used. [4]

Two-dimensional gel electrophoresis (2-DE) ways like two-dimensional polyacrylamide gel electrophoresis and two-dimensional distinction gel electrophoresis are fashionable techniques for proteins separation as a result of the permit researchers to characterize quantitative protein changes on an oversized scale. Thus, 2-DE is often used as an initial screening procedure whereby results obtained generate scientific concepts for study. These technologies revolutionized the sector of genetics and biomarker discovery in their ability to sight protein changes either in differential modification or expression [5]. The process of 2-dimensional gel electrophoresis is described below.

\subsection{Sample Preparation}

Effective sample preparation could be a key for the success of the experiment. The sample dictates the kind of extraction technique used, and therefore the solubility, charge, and $\mathrm{pl}$ of the proteome s of interest have an effect on the tactic of solubilisation. The proteome fraction used for 2-D action should be solubilised in a very denaturing answer of low ionic strength. This answer cannot contain elements that alter proteome size or charge. Sample preparation conjointly involves no obligatory steps to consume proteins, reduce the complexity of the protein mixture, or choose a sub proteome of interest.

In order to characterize specific proteins in a very advanced protein mixture, the proteins of interest should be fully soluble electrophoresis conditions. Totally different treatments and conditions are needed to solubilise differing types of protein samples; some proteins are naturally found in complexes with membranes, nucleic acids, or different proteins, some proteins type varied non-specific aggregates, and a few proteins precipitate when removed from their traditional surroundings. The effectiveness of solubilisation depends on the selection of cell disruption technique, protein concentration and dissolution technique, alternative of detergents, and composition of the sample answer. If any of those steps aren't optimized for a specific sample, separations are also incomplete or distorted and data is also lost [6].

\subsection{First-Dimension Isoelectric Focusing (IEF)}

The first-dimension separation of 2-D ionophoresis is IEF, wherever proteome s area unit separated on the idea of variations in their $\mathrm{pl}$. The $\mathrm{pl}$ of a proteome is that the hydrogen ion concentration at that it carries no internet charge, and it's a characteristic charged that's determined by the amount and kinds of charged teams the proteome carries.

Proteomes area unit amphoteric molecules that carry a negative, positive or zero net charge depending on $\mathrm{pH}$ of their atmosphere. For each protein, there is a selected $\mathrm{pH}$ at that its internet charge is zero. Proteome s show hefty variable in $\mathrm{pl}$, although $\mathrm{pl}$ values sometimes fall within the vary of ph3-12, with the bulk falling between $\mathrm{pH}-4$ and $\mathrm{pH}-8$. A protein is charged at hydrogen ion concentration values below its $\mathrm{pl}$ and charged at $\mathrm{pH}$ values higher than its $\mathrm{pl}[7]$.

For IEF, a protein is placed during a medium with a $\mathrm{pH}$ scale gradient and subjected to an electrical field. In response to the sphere, the protein moves toward the electrode with the alternative charge. On the other hand, it either picks up or loses protons. Its net charge and quality decrease till the protein eventually arrives at the purpose within the $\mathrm{pH}$ scale gradient adequate its $\mathrm{pl}$. There, the proteome is drained and stops migration. If by diffusion, it drifts off from the purpose within the gradient comparable to its $\mathrm{pl}$, it acquires charge and is force back. During this manner, proteins condense, or square measure centered, into sharp bands within the $\mathrm{pH}$ scale gradient at their characteristic pl values.

To fully analyze all living thing proteomes, the cells should be effectively discontinuous. Alternative of disruption technique depends on whether or not the sample is from cells, solid tissue, or different biological material and whether or not the analysis is targeting all proteins or simply a specific sub-cellular fraction. 
IEF return till a gradual state is reached. Proteins approach their $\mathrm{pl}$ values at a distinct rates however stay comparatively mounted at those $\mathrm{pH}$ scale values for extended periods. This can be in distinction to standard activity, wherever proteins still move through the medium till the electrical field is removed. In IEF, proteins migrate to their steady-state positions from anyplace within the system. IEF for 2-D activity is performed underneath denaturing conditions in order that proteins square measure utterly disaggregated and every one charged team's square measure exposed to the majority answer. Consequently, resolution is best underneath denaturing condition. Complete denaturation and solubilisation square measure needed to reduce aggregation and building block interactions, therefore making certain that every protein is gift in precisely one configuration.

\subsection{Second-Dimension SDS-PAGE}

SDS-PAGE (SDS-polyacrylamide gel electrophoresis) is electrophoresis methodology for separating polypeptides per their molecular weights. The intrinsic electrical charge of the sample proteins is not an element within the separation because of the presence of SDS within the sample and therefore the gel. SDS is associate degree anionic that, once in solution in water, forms globular micelles composed of 70-80 molecules with the dodecyl hydrocarb on moiety within the core and therefore the sulfate head teams within the hydrophilic shell. SDS and proteins form complexes with a necklace-like structure composed of proteome -decorated micelles connected by short versatile peptide segments [8]. When proteins square measure treated with each SDS and a reluctant, the degree electrophoresis separation among a polyacrylamide gel depends mostly on the relative molecular mass of the protein. In fact, there's associate degree around linear relationship between the index of the relative molecular mass and therefore the relative distance of migration of the SDS-polypeptide advanced [9].

The most unremarkably used buffer system for second-dimension SDS-PAGE is that the tris-glycine system represented by Laemmli [9]. This buffer system separates proteins at high $\mathrm{pH}$ scale that confers the advantage of lowest proteome aggregation and clean separation even at comparatively serious proteome masses. The Laemmli buffer system has the disadvantage of a restricted gel shelf life. Ettan DALT formed gels utilize a substitute buffer system supported piperidinopropionamide (PPA), which mixes long shelflife with the high separation $\mathrm{pH}$ scale of the Laemmli system. Alternative buffer systems can even be used, notably the Tristricine system of Schagger and von Jagow [10] for resolution of polypeptides within the Mr below ten 000. ExcelGel formed gels for second-dimension SDS-PAGE on the Multiphor II flatbed system utilize a distinct Tris-tricine buffer system.

\subsection{Detection}

In 2-D electrophoresis, proteins in gels square measure most typically visualized total protein stains. Alternative of the foremost acceptable stain involves thought of the stain uniqueness, limitations with relation to the sensitivity of detection and therefore the varieties of proteome s it stains best, downstream application, and therefore the form of imaging instrumentality accessible. To be used in proteomics applications. Stains ought to be compatible with high throughput protocols and downstream analysis, as well as digestion and mass spectrometry.

It is additionally attainable to label proteome samples preparation and before IEF with fluorescent dyes like the CyDye DIGE fluors. At the time of writing, three dyes with spectrally totally different excitation and emission wavelengths were accessible, permitting labelling of up to a few totally different samples and their separation during a single 2-D gel. The dyes square measure matching for size and charge to get migration of otherwise tagged samples square measure mixed along before they're applied on the gel of the primary dimension. Once separation, the gels square measure scanned with visible light imagers at the various wavelengths.

\subsection{Image Acquisition and Analysis}

2.5.1 Image Acquisition:In proteomic applications, selecting the image acquisition device depends on the staining technique used. A number of imaging systems are capable of multiple detection modes and can be used with a variety of applications.

Densitometers enable the visualization of gels stained with visible light-absorbing stains such as negative, coomassie or silver stains. Charge-couple device camera system can featured different light source for greater application flexibility. The can be used for visualization of visible and fluorescent stains and of chemiluminescence in some cases. Laser-based scanners offer the highest sensitivity, resolution.

2.5.2 Image Analysis: Spot detection is performed on the proteome map. As a result, a agreement spot pattern is generated, that is valid for all gels within the experiment. It describes the position and also the general form of all proteome spots from the experiment.

For spot division and building expression profiles, the agreement spot pattern is applied to any or all gel pictures of the experiment. The image transformation assures that each one spots of the agreement pattern attain 
their correct position. A remodelling step makes certain that the preset spot boundaries from the agreement area unit tailored to the important grey levels discovered on the target image. All boundaries of the agreement pattern will be found on each gel.

\section{I.5.3 Extracting Expression Profile:}

Expression profile analysis identifies fascinating spots which is able to be marked for any analysis, proteome identification, and interpretation [12]. For Extracting Expression Profile we had taken image in a text format. 2D volume data intensities as input, datasets must be exported from specialized Image Software in the form of a data frame of volume data $\mathrm{Xj}$; with gels $\mathrm{j}$ as columns and spots $\mathrm{i}$ as rows [12].

\begin{tabular}{|l|l|l|l|l|l|l|}
\hline \multirow{2}{*}{} & \multicolumn{3}{|l|}{ Replicates Condition 1 } & \multicolumn{3}{l|}{ Replicates Condition 1 } \\
\cline { 2 - 7 } & Gel1 & Gel2 & Gel3 & Gel1' & Gel2' & Gel3' \\
\hline Spot $_{1}$ & $\mathrm{X}_{1,1}$ & $\mathrm{X}_{2,1}$ & $\mathrm{X}_{3,1}$ & $\mathrm{X}_{1^{\prime}, 1}$ & $\mathrm{X}_{2}{ }^{\prime}, 1$ & $\mathrm{X}_{3^{\prime}, 1}$ \\
\hline Spot $_{2}$ & $\mathrm{X}_{1,2}$ & $\mathrm{X}_{2,2}$ & $\mathrm{X}_{3,2}$ & $\mathrm{X}_{1^{\prime}, 2}$ & $\mathrm{X}_{2,2}$ & $\mathrm{X}_{3^{\prime}, 2}$ \\
\hline Spot $_{i}$ & $\mathrm{X}_{1, \mathrm{i}}$ & $\mathrm{X}_{2, \mathrm{i}}$ & $\mathrm{X}_{3, \mathrm{i}}$ & $\mathrm{X}_{1^{\prime}, \mathrm{i}}$ & $\mathrm{X}_{2,, i}$ & $\mathrm{X}_{3^{\prime}, \mathrm{i}}$ \\
\hline
\end{tabular}

Table 1: Example of input data

Note that the name of columns should therefore correspond to the names of the gels and the names of the rows to the name of the spots. The replicates for each condition should be ordered in the following columns (see Table 1). Furthermore, another data frame is needed to describe the experiment with the names of gels as row names and a single column giving the two level of condition for data. On that text file following steps are performed:

\section{I.5.3.1 Visualization: It consists in plotting the intensity $\log 2$-ratio (R) against mean $\log 10$ intensity (I):}

$$
R=\log _{2} \frac{\operatorname{mean}\left(V_{\text {Cond } 2}\right)}{\text { mean }\left(V_{\text {Cond } 1}\right)}
$$

The intensity (I) is the $\log 10$ of the mean of volume data in condition 2 by the mean of volume data in condition 1 :

$$
I=\log _{10}\left(\text { mean }\left(V_{\text {Cond } 2}\right) \times \operatorname{mean}\left(V_{\text {Cond1 }}\right)\right)
$$

Where $\mathrm{V}_{\text {Cond1 }}$ and $\mathrm{V}_{\text {Cond2 } 2}$ are spot volumes for conditions 1 and 2, respectively [13].

I.5.3.2 Normalization: 2D Gel Volume data must be normalized in order to remove systemic variation prior to data analysis. Two widely used methods are provided, the "Variance Stabilizing Normalization" (vsn) and the "Quantiles, Normalization". The principle of the "quantiles normalization" is to set each quantile of each column (i.e. the spots volume data of each gels) to the mean of that quintile across gels. The intention is to make all the normalized columns have the same empirical distribution. Whereas the vsn methods relies on a transformation $h$, of the parametric form $h(x)=\operatorname{arsinh}(a+b x)$. The parameters of $\mathrm{h}$ together with those of the calibration between experiments are estimated with a robust variant of maximum-likelihood estimation. Both methods re-entered the data around a zero log ratio, nevertheless for low values of intensities the vsn normalized data seems to be less efficient in order to re-entered the cloud of points [13].

I.5.3.3 Coerce data into an ExpressionSet: Prior to analysis for differentially expressed proteins, data must be coerced into an ExpressionSet. This can be done easily with ES.prot, which requires a matrix of normalized volume data, the number of replicates in each condition and a dataframe giving the condition for the experiment. The matrix of spots intensities (i.e. Volume) is $\log 2$ transformed and stored in the assayData slot of the ExpressionSet. Furthermore, the log2-ratio is computed and stored in the featureData slot [13].

I.5.3.4 Find differentially expressed proteins: 2-DE experiments analysis require a variant of the t-statistic that is suitable for high-dimensional data and large-scale multiple testing. For that following steps are used:

i. the classical Student's t-test.

ii. two tests especially modified for micro-array analysis.

iii. two methods that take advantage of hierarchical Bayes methods for estimation of the variance across genes.

As statistical tests allowing the identification of differentially expressed proteins must take into account a correction for multiple tests in order to avoid false conclusions. There are different methods to estimate the False Discovery Rate : 
i. $\quad$ the classical FDR estimator of Benjamini and Hochberg.

ii. the local FDR estimator of Strimmer.

iii. the "robust FDR" estimator of Pounds and Cheng.

I.5.3.5 Simulation of 2D Volume data: In order to compare FDR and the responses of the different tests as well as the influence of the number of replicates, simulated data be used [13].

\subsection{What is Mass Spectrometry?}

\section{MASS SPECTROMETRY}

Mass Spectrometer is a quantitative tool used to measure the mass-to-charge ratio of ions. The two primary methods for ionization of whole proteins are electrospray ionization (ESI-which turns the sample proteins into ions) and matrix-assisted laser desorption/ionization (MALDI- It uses laser to ionize the sample proteins and then push the proteins into the analyzer to produce a Mass Spectrum.).

A typical Mass Spectrometer consists of three parts: a mass analyzer, a detector and an ion source. The ion source produces ions from the sample. The Mass Analyzer separates ions with different mass-to-charge ratios, these different ions are detected by detector. Finally, the mass spectrum is generated after all the data have been collected.

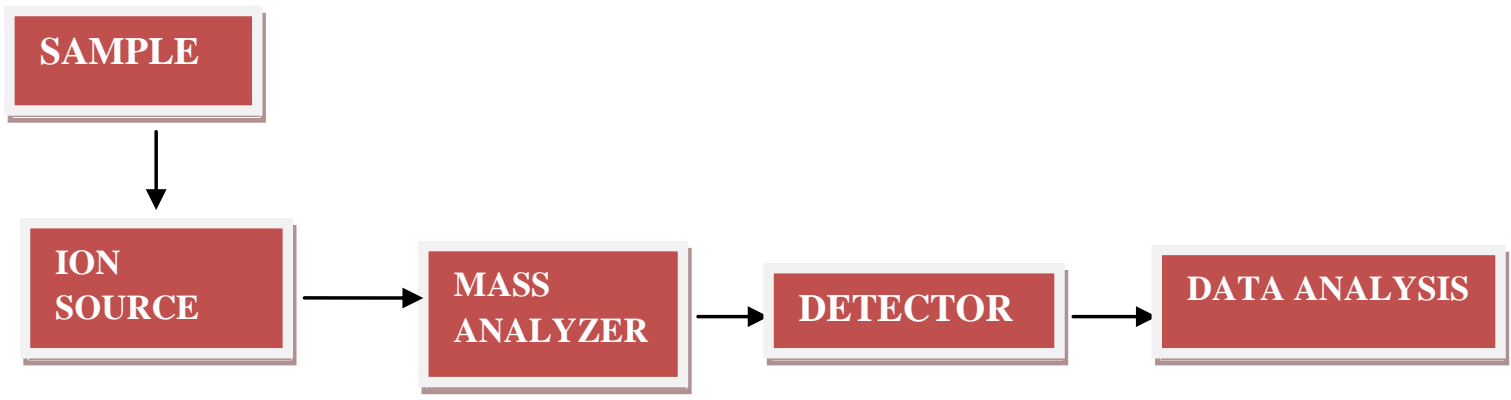

FIG 1: Scheme Graph of Mass Spectrometry

The history of mass spectrometry has its growth lies in physical and chemical research regarding the properties of matter. The research carried out on gas discharges in the mid 19th century led to the findings of anode and cathode rays, which results into positive ions and negative ions. In the early era, many professional scientists were concentrated around with electricity flowing through vacuum tubes. They were flooding the electrons from cathode (-ve) to anode (+ve) which Eugen Goldstein coined as "cathode rays".

\subsection{Data Analysis}

Mass spectrometry data analysis particular to the type of experiment producing the data. Fundamental divisions of data are general in understanding any data. Many mass spectrometers work in either electron mode or positive ion mode. Sometimes it is very necessary to know whether the obtained ions are negatively or positively charged. It is often essential in determining the neutral mass but it also symbolizes something about the nature of the molecules.

Various ion source results in arrays of fragments obtained from the fundamental molecules. The ionization source creates many fragments and mostly single-charged (1-) radicals (odd number of electrons), whereas nonradical quasimolecular ions, an electrospray source usually produces that are frequently multiply charged. Tandem mass spectrometry produces fragment ions post-source and can change the kind of data obtained by an experiment.

A strongly prepared biological sample will probably contain a specific amount of salt, adducts get formed with the analyte molecules in various analyses Knowledge of the initial sample can provide insight into the component molecules of the sample and their peptides(protein in form of fragments). A sample from this synthesis/manufacturing process will probably consist of impurities chemically related to the target component.

Results can also depend heavily on sample preparation and how it was introduced. Much of the energetics of the desorption/ionization event is controlled by the matrix rather than the laser power. Mass spectrometry can measure sample purity, molecular structure and molar mass. Each of these queries requires a different experimental procedure; so adequate definition of the experimental goal is an early requirement for collecting the proper data and evolving the data. 


\subsection{Interpretation of mass spectra}

Since the specific structure or peptide sequence of a molecule is decrypted through the set of fragment of masses, the combined usage of various techniques helps in the interpretation of mass spectra. The first way of identifying an unknown compound is to compare its experimental mass spectrum against databases of mass spectrum. The software assisted interpretation or manual interpretation of mass spectra must be performed if match in spectral database in not found. Computer simulation of ionization and fragmentation processes occurring in mass spectrometer is the fundamental tool for assigning structure or peptide sequence to a molecule. Such simulation is often supported by a fragmentation library that contains published patterns of known decomposition reactions. Software developed on this idea has been developed for both small molecules and proteins.

\subsection{Mass spectrometry data analysis}

After receiving the MS data, it has to go through various steps as follows[20]:

Data Storage and Security: Due to large size of biological data sets, a storage solution will be implemented to provide redundant and scalable data storage.

Data transformation: to standardized proteomics data.

Spectral visualization: enables the user to manipulate data, using various data visualization functionalities.

Spectral deconvolution: to differentiate signals arising from the real analyses as opposed to signals arising from contaminants or instrumental noise, and to reduce data dimensionality, to help in statistical analysis.

Peak alignment: recognizes peaks from the same molecule occurring in different samples from the millions of peaks detected during the course of an experiment.

Data normalization: allows multi-experiment analyses. It is important to first normalize the data for sample comparison. Normalization attempts to quantitatively filter overall peak intensity variations due to experimental errors.

Statistical testing: identify peptide or metabolite peaks that either make significant contributions to the molecular profile of a sample or distinguish a group of samples from others.

Pattern recognition: to recognize the particular pattern in which data is generated.

\subsection{Advantages and Disadvantages of Mass Spectrometry}

a. Advantages:

i) High Sensitivity-ability to detect very small amounts)

ii) High Selectivity- Ability to tell molecules apart in a mixture

iii) High Time Resolution

iv) Low Cost

v) small sample size

vi) fast

vii) differentiates isotopes

viii) can be combined with GC and LC to run mixtures, or can also be run in tandem for proteins or peptides etc.

b. Disadvantages:

i) doesn't directly give structural information (although we can often figure it out)

ii) Needs pure compounds.

iii) Difficult with non-volatile compounds.

\subsection{Application of Mass Spectrometry}

Mass Spectrometry consists of determining the isotopic composition of elements in a molecule, identifying unknown compounds, and determining the structure of a compound by understanding its fragmentation. Other uses include evaluating the amount of a compound in a sample or studying the fundamentals of gas phase in 
chemistry MS is now in very common use in analytical laboratories that study chemical, physical, or biological properties of a great variety of compounds.

\section{A.Isotope ratio MS: isotope dating and tracking}

Mass spectrometry is also used to determine the composition of elements in a sample. Variations in mass among isotopes of an element are very less, and the less abundant isotopes of an element are typically very rare, so a very sensitive instrument is requirement of Mass Spectrometry. These instruments, sometimes allude to as isotope ratio mass spectrometers (IR-MS), it uses a single magnet to bend a beam of ionized particles towards a series of Faraday cups which convert particle impacts to electric current. Isotope ratios are important markers of a variety of processes. The age of materials for example as in carbon dating is determined by isotopes ratios. Marking with isotopes which are stable are also used for protein quantification.

\section{B.Atom probe}

An atom probe is an instrument that combines field ion microscopy (FIM) time-of-flight and mass spectrometry to map the location of individual atoms.

\section{C.Pharmacokinetics}

Pharmacokinetics is often studied using mass spectrometry because of the complex nature of the matrix (often blood or urine) and the need for high sensitivity to observe low dose and long time. With a triple quadruple mass spectrometer, LC-MS is the most easily accessible instrumentation used; Tandem mass spectrometry is usually used for added probabilty. For quantization of usually a single pharmaceutical in the samples, standard curves and internal standards are used. The samples represent different time points as a pharmaceutical is administered and then metabolized or cleared from the body. There is currently considerable interest in the use of very high sensitivity mass spectrometry for which is a promising alternative as micro dosing studies, to animal experimentation.

\section{COMPARISON OF TWO TECHNIQUES}

We will see the various level comparison [21] of the two techniques discussed above:

\begin{tabular}{|c|c|c|c|}
\hline Sr no & Description & 2-D gel Electrophoresis & High Throughput Mass Spectrometry \\
\hline \multirow[t]{2}{*}{1.} & Method description & $\begin{array}{l}\text { - Separation of complex proteins via 2D gel } \\
\text { electrophoresis based charge and size }\end{array}$ & $\begin{array}{l}\text { - LC to separate proteins in a sample, with } \\
\text { sequential LC for improved separation } \\
\text { efficiency }\end{array}$ \\
\hline & & $\begin{array}{l}\text { - Major protein identification by MS, Detects } \\
\text { about } 2000-2500 \text { spots/gel }\end{array}$ & $\begin{array}{l}\text { - MS to systematically identify the major } \\
\text { proteins, Detects over } 1000 \text { proteins/run }\end{array}$ \\
\hline \multirow[t]{3}{*}{2.} & Advantages & - Ability to identify unknown proteins & - Ability to identify unknown proteins \\
\hline & & $\begin{array}{lll}\text { Detects protein } & \text { modification } \\
\text { (phosphorylation and methylation) }\end{array}$ & $\begin{array}{l}\text { - improved separation efficiency compared to } \\
\text { 2D gel }\end{array}$ \\
\hline & & $\begin{array}{l}\text { Used for various biological samples, including } \\
\text { tissue, blood and other biological fluids }\end{array}$ & $\begin{array}{l}\text { Used for various biological samples, including } \\
\text { tissue, blood and other biological fluids }\end{array}$ \\
\hline \multirow[t]{4}{*}{3.} & Disadvantages & $\begin{array}{l}\text { - Proteins expressed at low abundance may be } \\
\text { missed }\end{array}$ & $\begin{array}{l}\text { - Proteins expressed at low abundance may be } \\
\text { missed }\end{array}$ \\
\hline & & - Unsuited for diagnostic application & - Unsuited for diagnostic application \\
\hline & & $\begin{array}{l}\text { - Limited reproducibility and high rate of false } \\
\text { identification }\end{array}$ & $\begin{array}{l}\text { - Limited reproducibility and high rate of false } \\
\text { identification }\end{array}$ \\
\hline & & $\begin{array}{l}\text { Limited } \\
\text { range }\end{array}$ & $\begin{array}{l}\text { Limited } \\
\text { range }\end{array}$ \\
\hline \multirow[t]{2}{*}{4.} & Sensitivity & $\begin{array}{l}\text { - Detection sensitivity is in the nanogram } \\
\text { range ( } 50 \mathrm{ng} / \mathrm{spot} \text { for Coomassie Blue; } 1 \\
\text { ng/spot for silver stain) }\end{array}$ & $\begin{array}{l}\text { - Detection sensitivity is in the nanogram range } \\
\text { or } \sim 20 \text { cells }\end{array}$ \\
\hline & & $\begin{array}{l}\text { Using fluorescent 2D-differential gel } \\
\text { electrophoresis ( } 2 \mathrm{D} \text {-DIGE), sensitivity improves } \\
\text { by } 10 \text { fold (CyDye label) }\end{array}$ & $1 \%$ false positive rate \\
\hline
\end{tabular}

Table 2: Comparisons between 2-D gel Electrophoresis and Mass Spectrometry

\section{CONCLUSION}

The data originated in the field of Proteomics is very large .It's the need of the day that these huge amount of data need to be managed and analyzed. Various tools and techniques are available for the protein prediction. 2-D gel Electrophoresis and Mass Spectrometry are the analytical method for data analysis which originated in large amount. We have seen the various aspects of 2-D Gel and Mass Spectrometry. Also we have seen the advantages and disadvantages of the methods and various applications of them. Through the paper, we can learn the current technologies of proteomics. 


\section{REFERENCES}

[1] Wilkins M. R., Sanchez J.-Cooley A. A., et al. "Progress with proteome projects: why all proteins expressed by a genome should be identified and how to do it". Biotechnology and Genetic Engineering Reviews, vol. 13 pp. 19-50 1995 2-s2.0-0030333694.

[2] O'Farrell, P.H. High resolution two-dimensional electrophoresis of proteins. J. Biol. Chem. 250, 4007-4021 (1975).

[3] Klose, J. Protein mapping by combined isoelectric focusing and electrophoresis of mouse tissues, A novel approach to testing for induced point mutation in mammals. Humangenetik 26, 231-243 (1975).

[4] IDTutorial: Gel Electrophoresis

[5] Kimberly F. Sellers, Jeffrey C. Miecznikowski," Statistical Analysis of Gel Electrophoresis Data".

[6] "2-D Electrophoresis using immobilized $\mathrm{pH}$ gradients- Principles and Methods", Amersham Biosciences.

[7] “2-D Electrophoresis workflow”, fourth edition, BIO-RAD.

[8] Ibel, K., May, R.P., Kirschner, K., Szadkowski, H., Mascher, E., Lundahl, P. Protein-decorated micelle structure of sodiumdodecyl-sulfate protein complexes as determined by neutron scattering.Eur. J. Biochem. 190,311-318 (1990).].

[9] Laemmli, U.K. Cleavage of structural proteins during the assembly of the head of bacteriophage T4.Nature 227, 680-685 (1970)

[10] Schagger, H., von Jagow, G. Tricine-sodium dodecyl sulfate-polyacrylamide gel electrophoresis for the separation of proteins in the range from 1 to $100 \mathrm{kDa}$. Anal. Biochem. 166, 368-379 (1987).

[11] Andrew W. Dowsey, Jeffrey S. Morris, Howard B. Gutstein, and Guang-Zhong Yang, "Informatics and Statistics for Analyzing 2-D Gel Electrophoresis Images", NIH Public Access Author Manuscript Methods Mol Biol. Author manuscript; available in PMC, 2013.

[12] Matthias Berth \& Frank Michael Moser \& Markus Kolbe \& Jorg Bernhardt," The state of the art in the analysis of two-dimensional gel electrophoresis images", Received: 10 May 2007 /Revised: 13 July 2007 /Accepted: 14 July 2007 /Published online: 23 August 2007 \# Springer-Verlag, 2007.

[13] S'ebastien Artigaud,"prot2D: Statistical Tools for volume data from 2D Gel Electrophoresis".

[14] Pandey, A., \& Mann, M. (2000), "Proteomics to study genes and genomes.", Nature, Vol. 405, No. 6788, pp. 837-846.

[15] Bro, C., \& Nielsen, J., (2004), "Impact of 'ome' analyses on inverse metabolic engineering. “, Metabolic Engineering, Vol. 6, No. 3, pp. 204-211.

[16] Wilkins, M.R., Pasquali, C., Appel, R.D., Ou, K., Golaz, O., Sanchez, J.C., Yan, J.X., Gooley, A.A., Hughes, G., Humphery-Smith, I., Williams, K.L., \& Hochstrasser, D.F., (1996a), "From proteins to proteomes: large scale protein identification by twodimensional electrophoresis and arnino acid analysis.", Nature Biotechnology,Vol. 14, No. 1, pp. 61-65.

[17] Fatemeh Tabandeh, Parvin Shariati and Mahvash Khodabandeh, "Application of Two-Dimensional Gel Electrophoresis to Microbial Systems", National Institute of Genetic Engineering and Biotechnology (NIGEB), Tehran, Iran.

[18] Haleem J.Issaq and Timothy D.Veenstra, "Two-Dimensional polyacrylamide gel electrophoresis (2D-PAGE): advances and perspectives", BioTechniques 44:697-700 (25 "Anniversary Issue, April 2008) doi 10.2144/000112823.

[19] Weston, Andrea D.; Hood, Leroy (2004). "Systems Biology, Proteomics, and the Future of Health Care: Toward Predictive, Preventative, and Personalized Medicine". Journal of Proteome Research 3 (2): 179-96.

[20] Catherine P. Riley*, Erik S. Gough, Jing He, Shrinivas S. Jandhyala, Brad Kennedy, Seza Orcun, Mourad Ouzzani, Charles Buck, Ali M. Roumani and Xiang Zhang, " The Proteome Discovery Pipeline - A Data Analysis Pipeline for Mass Spectrometry-Based Differential Proteomics Discovery": The Open Proteomics Journal, 2010, vol 3, pg[8-19]

[21] Comparison of different proteomics-based- an additional file.

[22] Jimmy K. Eng, Ashley L. McCormack, and John R. Yates, III (1994). "An Approach to Correlate Tandem Mass Spectral Data of Peptides with Amino Acid Sequences in a Protein Database". J Am Soc Mass Spectrom 5 (11): 976-989

[23] Perkins, David N.; Pappin, Darryl J. C.; Creasy, David M.; Cottrell, John S. (1999). "Probability-based protein identification by searching sequence databases using mass spectrometry data".Electrophoresis 20 (18): 3551-67 